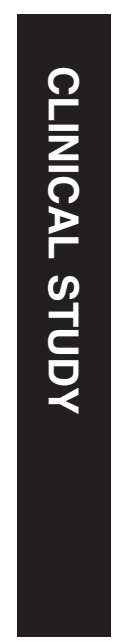

\section{Portsmouth visual field database: an audit of glaucoma progression}

${ }^{1}$ Department of Ophthalmology, Queen Alexandra Hospital, Portsmouth, UK

${ }^{2}$ Department of Optometry and Visual Science, City University London, London, UK

${ }^{3}$ Ophthalmology

Department, Gloucestershire Hospitals NHS Foundation Trust, Cheltenham General Hospital, Cheltenham, UK

Correspondence:

JF Kirwan, Queen

Alexandra Hospital, Cosham, Portsmouth PO6 3LY, UK

Tel: +44 239228 6000;

Fax: +44 2392286933

E-mail: jfkirwan@mac.com

Received: 17 June 2013 Accepted in revised form: 11 November 2013 Published online: 30 May 2014

Part of this work has been previously presented at the United Kingdom and Eire Glaucoma Society Annual Meeting 2011, Manchester, UK.

\begin{abstract}
Aim To explore visual field (VF) progression in a cohort of secondary care-treated glaucoma and ocular hypertensive (OHT) patients. Methods We extracted VFs from our database drawn from our normal clinical practice. VF series from 4177 eyes from 2208 patients who had five or more VFs were obtained, the 'better' eye was selected and the rate of VF progression was calculated using mean deviation (MD) data. Results The median rate of progression for the whole sample was $-0.1 \mathrm{~dB} /$ year (interquartile range (IQR) -4 to $0 \mathrm{~dB} /$ year) over a median of 6.7 years (IQR 4.9-8.7). Of 2208 patients, $477(21.2 \%)$ progressed at $>-0.5 \mathrm{~dB} /$ year; $46(2.1 \%)$ progressed at $>-2.0 \mathrm{~dB} /$ year. Of those with a 'final MD' of worse than $-10 \mathrm{~dB}(N=244)$ in their better eye; $14.0 \%$ were 'fast progressors' ( $>-2 \mathrm{~dB} /$ year), $33.7 \%$ 'moderate progressors' ( -1 to $-2 \mathrm{~dB} /$ year), and $28.8 \%$ 'slow progressors' ( $-0.3 \mathrm{~dB}$ to $-1 \mathrm{~dB} /$ year). Of those with 'initial MD' better than $-3 \mathrm{~dB}$ and those with worse than $-3 \mathrm{~dB}, 31 / 1679$ $(1.8 \%)$ and $213 / 529(40.3 \%)$ respectively, had a final MD of worse than $-10 \mathrm{~dB}$. Conclusion Fast progressors, while important, are relatively rare. Moderate and slow progressors make up the majority of the progressing population within this data set. The risk of significant visual loss is much higher in those with initial damage. With increasing life expectancy, moderate and slow progressors may become increasingly clinically important.

Eye (2014) 28, 974-979; doi:10.1038/eye.2013.294; published online 30 May 2014

Keywords: ocular hypertension; glaucoma; epidemiology; disease progression (MeSH terms)
\end{abstract}

JF Kirwan' ${ }^{1}$, A Hustler ${ }^{1}$, H Bobat ${ }^{1}$, L Toms ${ }^{1}$, DP $\mathrm{Crabb}^{2}$ and Al McNaught ${ }^{3}$

Introduction

Open angle glaucoma is a chronic, progressive optic neuropathy, with characteristic morphological changes at the optic nerve head and retinal nerve fibre layer associated with characteristic visual field (VF) loss. ${ }^{1}$ Standard automated perimetry (SAP) is the most common method for assessing VF in glaucoma and has been in wide use for many years. ${ }^{2,3}$ There is relatively little known about the natural history of VF progression in treated glaucoma patients in secondary care. ${ }^{4}$ There are descriptive studies of large populations in tertiary care settings, but these may not represent glaucoma in a broader setting. ${ }^{5-9}$ Portsmouth Hospitals NHS Trust serves a population of $\sim 550$ to 600000 ; over $95 \%$ of the population are Caucasian.

The hospital covers the majority of the local population which is well defined and relatively stable; a very small proportion $(<5 \%)$ is seen by other health-care providers. ${ }^{10}$

Clinical practice relies upon serial VF measurements to detect disease progression. Software packages for detecting VF progression include Statpac (Carl Zeiss Meditec, Dublin, CA, USA) and PROGRESSOR (Medisoft Ltd, Leeds, UK). These have been shown to aid more consistent clinical decisions about progression. ${ }^{11-14}$ We have used the same VF testing strategy, Swedish Interactive Threshold Algorithm (SITA) Fast 24-2 (Carl Zeiss), for the last 14 years, enabling us to measure a change in mean deviation (MD) over time. MD is a summary measure of a subject's overall VF sensitivity compared with age-related norms. Monitoring MD against time is a simple way of summarising the overall rate of VF loss in individuals ( $\mathrm{dB} /$ year) ${ }^{2,15}$ We have performed over 100000 Humphrey 'SITA Fast' 24-2 VF tests, 
with 45000 patients having at least one VF test. We report data on a cohort of secondary care-treated 'glaucoma and ocular hypertension (OHT)' patients who have had five or more VF tests to assess the distribution of rates of VF loss.

\section{Materials and methods}

We used the PROGRESSOR software to search the Portsmouth VF database for patients with five or more SITA Fast VFs. All patients had undergone VF assessment with the SITA fast 24-2 algorithm with the Humphrey Field Analyzer (HFA). Details of each patient identified including age, eye/eyes involved, the date and $\mathrm{MD}$ (in $\mathrm{dB}$ ) of each VF were then exported to the statistical software (SPSS Statistics v 19; IBM Inc., Redmond, WA, USA) for analysis. Each patient had either one or two eyes with five or more VFs; for patients with two eyes, data for each eye were extracted and dealt with as a separate sequence. We chose subjects with five or more VF tests to allow sufficient fields for an estimate of VF progression. To estimate rates of progression, we used a 'best' / 'best' analysis, whereby the least negative of the last two MDs 'final MD' (FMD) was subtracted from the least negative of the first two MDs, 'initial MD' (IMD) and dividing this by the time from the first to the penultimate VF test. To verify our assumption that patients having regular VF testing have glaucoma or OHT, we performed a 'validation' study, extracting case notes for a subsample of patients. We stratified patients into fast ( $>2 \mathrm{~dB} /$ year), medium, slow (0.5-2 dB/year), and non-progressors ( $<0.5 \mathrm{~dB} /$ year). We randomly sampled cases in each stratum and checked the case notes to verify diagnosis. Diagnosis was based on the clinical impression of the case notes and inspection of VF printout. If there was doubt, then the lead author (JFK) confirmed diagnosis. Where diagnosis changed, for example, from OHT or 'glaucoma suspect' to primary open angle glaucoma (POAG), the latter was used. For patients with asymmetric disease, for example, suspect glaucoma in one eye and definite POAG in the other eye, diagnosis was on a 'patient basis', that is, diagnosed as POAG.

\section{Results}

VFs recorded over the period October 1999 to December 2010 were available in the database (134 months), yielding 4177 eyes from 2208 patients that had five or more VFs. For each patient, the 'better eye' (defined by the least negative MD from the first pair of VFs) was selected. For the 239 patients with only one eye in the database, this eye was taken to be the better eye. All further analyses were performed on the 2208 individual patients' better eyes unless explicitly stated otherwise. Baseline data are summarised in Table 1.

\section{Presenting VF}

Median IMD value for the 2208 better eyes was $-0.9 \mathrm{~dB}$, interquartile range (IQR) -2.9 to 0.3 . Of the 2208 eyes, $1679(76 \%)$ had an IMD of better than $-3 \mathrm{~dB}, 1883 / 2208$ $(85.3 \%)$ had an IMD of better than $-5 \mathrm{~dB}$. There were $123 / 2208$ subjects $(5.6 \%)$ with an IMD of worse than $-10 \mathrm{~dB}$ at presentation.

\section{Rate of progression}

The median rate of progression for the cohort of better eyes was $-0.1 \mathrm{~dB} /$ year, (IQR -0.4 to $0 \mathrm{~dB} /$ year). Subgroups of the 2208 better eyes defined by the presenting and final MDs were defined, were analysed, and summarised in Table 2.

Patients were divided into groups by the rate of progression. The group progressing at more than $-2 \mathrm{~dB} /$ year was deemed 'fast progressors' (ie, the MD was decreasing by more than $2 \mathrm{~dB}$ /year).

\section{How initial degree of field loss relates to final field loss and progression rate}

To explore how the severity of the IMD compared with the severity of the FMD, we divided up our data by severity of IMD. This is shown in Table 3. Similarly, Table 4 shows the distribution of progression rates for a given degree of IMD.

\section{Validation study}

The records of 250 patients were selected for review. Records were available for 220 patients. The most common diagnoses were POAG $n=143$, normal-tension glaucoma (NTG) $n=19$, OHT $n=39$, pseudoexfoliation glaucoma (PXG) $n=2$, angle closure glaucoma $n=6$, and secondary glaucoma $n=10$. One case had a stable meningioma. For patients with an IMD of better than $-3 \mathrm{~dB}$ and no progression $(n=59), 36$ had OHT $(61 \%)$ and 19 had POAG (32\%).

\section{Discussion}

We have presented the VF progression rates in the better eye of treated 'glaucoma or $\mathrm{OHT}^{\prime}$ patients in a secondary care setting. This gives us an indication of the burden of disease from the 'treated' population (some may have been merely observed). It has been estimated that the mean age-related VF decline in non-glaucoma patients is $-0.06 \mathrm{~dB} /$ year. $^{16}$ Median rate of progression for our 
whole cohort was $-0.15 \mathrm{~dB} /$ year (mean $-0.27 \mathrm{~dB} /$ year), over a median of 6.7 years. Despite the slow overall rate of progression, a significant proportion of treated patients does progress to significant visual loss (MD $<-15 \mathrm{~dB}$ ). This demonstrates how a central estimate such as a median or a mean may not give useful summary information for these data. Of 2208 patients, $477(21.6 \%)$ progressed faster than $-0.5 \mathrm{~dB} /$ year, of whom $46(2.1 \%)$ progressed at $>-2.0 \mathrm{~dB} /$ year. We found that patients with significant VF loss at the beginning were more likely to progress at a fast rate. While for the whole cohort, $2.1 \%$ were fast progressors; for those with an initial field defect (IMD worse than $-3 \mathrm{~dB}$ ), the corresponding figures were $36.2 \%$

progressing at more than $-0.5 \mathrm{~dB} /$ year and $5.7 \%$ progressing at more than $-2 \mathrm{~dB} /$ year.

For patients with an IMD of better than $-3 \mathrm{~dB}, 13.2 \%$ had MD loss of greater than $-3 \mathrm{~dB}$ over the period of the study.

Table 1 Subject demographics

\begin{tabular}{ll}
\hline Median age at start of follow-up & 67.3 years (IQR 60.7-75.6) \\
Median age at end of follow-up & 75.0 years (IQR 67.7-82.2) \\
Median number of VF tests & 6 (IQR 5-8) \\
Median duration of follow-up & 6.7 years (IQR 4.9-8.7) \\
Proportion of patients with both eyes included & $1969 / 2208(89 \%)$ \\
Best eye right & $1174 / 2208(53 \%)$ \\
Median presenting MD best eye & $-2.0 \mathrm{~dB}(\mathrm{IQR}-2.9$ to 0.3$)$ \\
Median presenting MD worst eye & $-3.2 \mathrm{~dB}(\mathrm{IQR}-7.3$ to -0.9$)$ \\
\hline
\end{tabular}

${ }^{a}$ Negatively skewed distribution.

Table 2 Table showing distribution of progression rates

\begin{tabular}{lccccc}
\hline $\begin{array}{l}\text { Progression } \\
\text { (dB/year) }\end{array}$ & $\begin{array}{r}\text { Best } \\
\text { eyes } \\
(\%)\end{array}$ & $\begin{array}{c}\text { Initial }^{\mathrm{a}} M D \\
<-3 d B(\%)\end{array}$ & $\begin{array}{c}\text { Final }^{\mathrm{b}} M D \\
<-10 d B \\
(\%)\end{array}$ & $\begin{array}{c}\text { Final }^{\mathrm{b}} M D \\
<-15 d B \\
(\%)\end{array}$ & $\begin{array}{c}\text { All } \\
\text { eyes } \\
(\%)\end{array}$ \\
\hline$>-2$ & 2.1 & 5.7 & 14.0 & 23.0 & 2.7 \\
-2 to -1 & 7.3 & 12.7 & 33.7 & 28.0 & 8.0 \\
-0.3 to -1 & 22.5 & 29.7 & 29.4 & 32.0 & 25.4 \\
$>-0.3$ & 65.9 & 51.9 & 23.5 & 17.0 & 63.9 \\
Number of patients & 2208 & 528 & 243 & 100 & 4177 \\
\hline
\end{tabular}

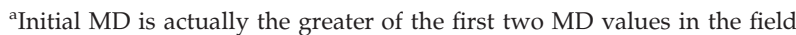
series.

${ }^{b}$ Final MD is actually the greater of the last two MD values in the field series.
In comparison, for patients with IMD worse than $-3 \mathrm{~dB}$, $31.4 \%$ had MD loss of more than $3 \mathrm{~dB}$.

The 1386 patients with an FMD of better than $-3 \mathrm{~dB}$ could reasonably be considered as OHT, pre-perimetric glaucoma cases, glaucoma suspects or patients with asymmetric glaucoma (since the fellow eye might be worse). Median deterioration for this group was $-0.05 \mathrm{~dB} /$ year.

Table 3 shows the distribution of FMD stratified by initial VF loss. Some subjects showed apparent improvement, this is probably due to a number of factors; first, for some patients, especially those with a relatively small number of fields, the variability in field tests in a 'stable' patient may give the appearance of improvement. Second, patients may have had cataract surgery. Third, perimetric learning may have occurred. ${ }^{17}$

A number of observations can be drawn from the data. First, only $8.8 \%$ of patients without a significant initial field defect (better than $-3 \mathrm{~dB}$ ) showed progression to an FMD of worse than $-5 \mathrm{~dB}$. In Table 4 , the proportion of fast and moderate progressors was higher in the groups with more advanced initial field loss.

We also looked at VF loss at the end of the study. One hundred patients had an FMD of worse than $-15 \mathrm{~dB}$ in their better eye, and 243 had an FMD of worse than $-10 \mathrm{~dB}$. Patients with this degree of VF loss in their better eye are very likely to have a degree of VF loss that renders them unable to pass the Driver and Vehicle Licensing Authority (DVLA) VF requirements. ${ }^{18}$ Of those patients who ended up at $-10 \mathrm{~dB}$, or worse, in their better eye $14.0 \%$ were 'fast progressors' (faster than $-2 \mathrm{~dB} /$ year), $33.7 \%$ progressed at a rate of $-1.0 \mathrm{~dB}$ to $-2 \mathrm{~dB}$ and $29.4 \%$ progressed at a rate of between -0.3 and $-1 \mathrm{~dB} /$ year; $23.5 \%$ were not progressing during the study. Slow rates of progression make up a large proportion of this population. With increasing life expectancy these moderate and slow progressors may become increasingly clinically important. Moderate and slow progression is harder to detect than fast progression. $^{2}$

This is a large retrospective clinic-based study, representative of clinical practice in secondary care in a

Table 3 Table showing distribution of 'final' MD values for a given degree of 'initial' MD

\begin{tabular}{lccccc}
\hline Initial $M D^{\mathrm{a}}(d B)$ & $\begin{array}{c}\text { N. with this } \\
\text { initial } M D\end{array}$ & $\begin{array}{c}\text { N. with final } \\
M D<-5 d B\end{array}$ & $\begin{array}{c}\text { N. with final } \\
M D<-7 d B\end{array}$ & $\begin{array}{c}\text { N. with final } \\
M D<-10 d B\end{array}$ & $\begin{array}{c}\text { N. with final } \\
M D<-15 d B\end{array}$ \\
\hline$>-3$ & 1679 & $147(8.8 \%)$ & $76(4.5 \%)$ & $31(1.8 \%)$ & $7(0.4 \%)$ \\
$<-3$ & 528 & $390(73.9 \%)$ & $304(57.6 \%)$ & $213(40.3 \%)$ & $95(18.0 \%)$ \\
$<-5$ & 318 & $289(90.9 \%)$ & $250(78.6 \%)$ & $189(59.4 \%)$ & $89(28.0 \%)$ \\
$<-7$ & 202 & $197(97.5 \%)$ & $191(94.4 \%)$ & $159(78.7 \%)$ & $80(39.6 \%)$ \\
$<-10$ & 122 & $121(99.2 \%)$ & $119(97.5 \%)$ & $114(93.4 \%)$ & $70(57.4 \%)$ \\
\hline
\end{tabular}

anitial MD is actually the greater of the first two MD values in the field series.

${ }^{\mathrm{b}}$ Final MD is actually the greater of the last two MD values in the field series. 
Table 4 Table showing distribution of progression rates for a given degree of 'initial' MD

\begin{tabular}{|c|c|c|c|c|c|}
\hline & & $\begin{array}{l}N . \text { with } \\
\text { progression } \\
\text { rate } \\
<-0.3 \mathrm{~dB} / \\
\text { year }\end{array}$ & $\begin{array}{c}N . \text { with } \\
\text { progression } \\
\text { rate }-0.3 \text { to } \\
-0.99 \mathrm{~dB} / \\
\text { year }\end{array}$ & $\begin{array}{c}N . \text { with } \\
\text { progression } \\
\text { rate }-1 \text { to } \\
-1.99 \mathrm{~dB} / \\
\text { year }\end{array}$ & $\begin{array}{c}\text { N. with } \\
\text { progression } \\
\text { rate } \\
>-2 d B / \\
\text { year }\end{array}$ \\
\hline $\begin{array}{l}\text { Initial } \\
M D^{\mathrm{a}}(d B)\end{array}$ & $\begin{array}{l}\text { N. with this } \\
\text { initial } M D\end{array}$ & $\begin{array}{c}\text { Non-prog } \\
\text { ressors }\end{array}$ & $\begin{array}{c}\text { Slow } \\
\text { progression }\end{array}$ & $\begin{array}{c}\text { Moderate } \\
\text { progression }\end{array}$ & $\begin{array}{c}\text { Fast } \\
\text { progression }\end{array}$ \\
\hline$>-3$ & 1679 & $1182(70.4 \%)$ & $383(22.8 \%)$ & $98(5.8 \%)$ & $16(1.0 \%)$ \\
\hline$<-3$ & 528 & $274(51.9 \%)$ & $157(29.7 \%)$ & $67(12.7 \%)$ & $30(5.7 \%)$ \\
\hline$<-5$ & 318 & $155(48.7 \%)$ & $93(29.2 \%)$ & $47(14.8 \%)$ & $23(7.2 \%)$ \\
\hline$<-7$ & 202 & $96(47.5 \%)$ & $59(29.2 \%)$ & $31(15.3 \%)$ & $16(7.9 \%)$ \\
\hline$<-10$ & 122 & $59(48.4 \%)$ & $40(32.8 \%)$ & $16(13.1 \%)$ & $7(5.7 \%)$ \\
\hline
\end{tabular}

${ }^{a}$ Initial MD is actually the greater of the first two MD values in the field series.

predominantly Caucasian population. As such we believe this is a reasonable representation of treated disease behaviour.

We chose the better eye to ensure that we would obtain the 'best-case' estimate of VF outcome, which would relate better to patient functioning. Problems with everyday visually dependent activities and quality of life are positively correlated with the level of vision in the better-seeing eye. ${ }^{19}$ Clearly, the overall progression rates would be greater if we looked at all eyes or if we had excluded those $1182(53.5 \%)$ patients that had an IMD of better than $-3 \mathrm{~dB}$ that did not progress (presumed OHT or stable glaucoma). However, we wished to include the whole cohort as well as the subsets with field loss or progression to reflect the overall burden. We used the simple 'best-best' approach to establish the rate of VF progression, because we felt that the 'better' VF tests were more likely to be a true reflection of the patient's VF. An alternative would have been linear regression.

However, with only five or six points for most subjects and a significant variability (especially for the first VF performed), we felt that our simpler approach would be reasonable. Because the first VFs in a series are often worse than second VFs, linear regression could give spuriously positive slopes as the early tests could be showing improvement due to the learning effect rather than any true changes. ${ }^{20}$

There are significant limitations to this retrospective data. Not all the VF data necessarily represent 'glaucoma' patients. There may be patients having sequential VF for other reasons such as a pituitary tumour (we found one such patient in our sample of 220 cases); however, the numbers will be very small and not materially affect our conclusions. It may be argued that only patients with reliable fields and acuities above a certain level should be included. However, patients may produce meaningful VFs but have one of the indices (particularly fixation) marked as unreliable. Including all patients may potentially increase 'noise', but can improve 'external validity' — by excluding patients, one may induce a degree of selection bias, making the data less representative of what we see in clinical practice. We believe that although there is potential for misclassification on an individual basis, the overall data are important and complementary to more precise, wellcharacterised data sets. ${ }^{5,21}$ Research data sets are often accrued in academic centers that may not be truly representative of the whole spectrum of diagnosed glaucoma in a population. In the future, with the development of 'big data', large validated data sets from routine clinical practice will be routinely available for ophthalmology as it is for general practice; with the uptake of electronic patient records and their long-term use. However, in the meantime, data such as ours may add useful information. ${ }^{22}$ Recording MD over time is simple but subject to flaws, particularly a lack of sensitivity, effect of other factors such as cataract and the absence of spatial information. However, for looking at trends in a population, these drawbacks are perhaps less important.

By including a minimum of five or more VF tests, we accept that some of the slope estimates are imprecise, but they do reflect the total number of VF tests a patient has in secondary care: only a subset of glaucoma patients had eight or more fields. We analysed this subset $(n=621)$ and found broadly similar results. There is literature showing that while not perfect, a linear model is reasonable to apply to both point-wise and MD data. ${ }^{13}$

Not all progression is due to glaucoma, other causes such as macular degeneration, cataract, retinal vein occlusion (RVO), or stroke will also have a role. $\mathrm{MD}$ values are influenced by the presence of lens opacities and by removal of cataract, which increased MD by $1.6 \mathrm{~dB}$ in one study. ${ }^{23}$ In our experience, patients who have sufficient cataract to cause a reduction in MD are likely to have cataract surgery. Whether the reduction in MD in those with progressing cataract, or the increase in MD with cataract surgery is unknown-they may well cancel each other out. Those with macular generation are unlikely to continue with VF testing if significant acuity is lost. If conditions such as RVO with a rapid onset occur, then they may erroneously increase the number of 'fast progressors' leading to an overestimate of their numbers.

The median rate of progression in the better eye for all our patients was $-0.15 \mathrm{~dB} /$ year; for those with an IMD of $<-3 \mathrm{~dB}$ (putative glaucoma patients), it was $-0.27 \mathrm{~dB} /$ year. Data from Sweden showed a faster rate of progression (median $0.62 \mathrm{~dB} /$ year). ${ }^{4}$ Possible reasons for the difference may be that we used data from the better eye and they used data from the eye with the larger VF defect. PXG is uncommon in our population 
but common in Sweden; PXG patients progress at a faster rate than POAG patients. ${ }^{24}$ The cases in the Swedish study included only those with definite glaucoma, their median initial MD was $-10 \mathrm{~dB}$.

A major factor that affects disease severity is the pattern of referral for glaucoma. In the United Kingdom, community optometrists detect most glaucoma patients and patients are encouraged to have regular eye tests. This means that many patients may present earlier than in other settings. Following the introduction of NICE guidelines in the United Kingdom, we have seen an increase in referrals that may have increased the yield of early glaucoma patients (albeit with many false positives). ${ }^{25}$

Both the Early Manifest Glaucoma Trial (EMGT) and the Collaborative Normal Tension Glaucoma Study (CNGTS) report the rate of VF progression in untreated glaucoma. In EMGT, the combined mean rate of VF progression was $-1.08 \mathrm{~dB} /$ year, the rate being higher in those with PXG ( $-3.13 \mathrm{~dB} /$ year). ${ }^{24}$ In CNGTS, the mean rate of VF progression was $-0.39 \mathrm{~dB} /$ year. $^{26}$

Previous studies of progression in definite glaucoma patients quote rates ranging from $-0.11 \mathrm{~dB}$ to $-0.45 \mathrm{~dB} /$ year. $^{27-30}$ We do not yet know how best to identify 'at-risk' patients reliably in routine clinical practice. In our study, patients with advanced field loss at presentation have a high risk of further field loss even under treatment, $37.9 \%$ of patients with an IMD of between -5 and $-10 \mathrm{~dB}$ on presentation will deteriorate to worse than $-10 \mathrm{~dB}$. A low proportion of subjects with minimal or early field loss progresses significantly but their numbers are large $(76 \%$ of our subjects) with consequent resource issues.

Detecting moderate rates of progression in the presence of long-term fluctuation in VF is difficult. Nouri-Mahdavi et $a l^{31}$ found that twice yearly VF testing resulted in earlier detection of glaucoma progression compared with yearly tests. Chauhan et al ${ }^{2}$ suggested that six VFs are performed in the first 2 years, to exclude rapid progression. $\mathrm{Crabb}^{32}$ has suggested using a 'wait and see' approach, where three VF tests are performed shortly after diagnosis, and three VF tests 2 years later; this may be more efficient. The focus of these recommendations on VF testing frequency is to detect rapid progressors, $>-2.0 \mathrm{~dB} /$ year; our data suggest that the majority of patients who lost vision in their better eye due to VF progression had less rapid rates of progression.

We noted that patients with initial VF damage are more likely than those without VF damage to progress to a significant visual disability. This has been found in some reports but not all. ${ }^{8,33}$ Patients with initial damage need frequent VFs, especially in early follow-up to establish their risk of progressing to blindness or severe visual disability in their lifetime. Those with normal or early changes, for example, with MD better than $-3 \mathrm{~dB}$, may not require such frequent $\mathrm{VF}$ testing. If all patients have six VFs in the first 2 years, then there may not be enough VF slots for those high-risk patients who present with initial VF changes, especially in health systems with some constraints as to the availability of VF testing slots. ${ }^{34}$

In the United Kingdom, there is a move towards glaucoma care being delivered through a number of independent providers within a single health-care district. Having access to a patient's full VF history is important to establish disease progression; if patient care is transferred between providers it is important that the full VF history transfer with the patient.

This large data set describes the rate of VF progression, in the better eye, of 'treated' 'glaucoma and OHT' patients, in secondary care. Patients with the worst initial VF damage were by far, at the greatest risk of visual disability and those with the fastest rate of VF loss were most commonly those with initial VF damage. It may be appropriate to reduce the VF resource given to OHT patients, particularly those with no other risk factors for glaucoma. With increasing life expectancy, the 'window of risk' is likely to increase and resources need to be appropriately focused on those patients at the highest risk of eventual visual disability.

\section{Summary}

What was known before

- Relatively little is known about the range and prevalence of different rates of VF progression in treated 'glaucoma and $\mathrm{OHT}^{\prime}$ patients in secondary care.

\section{What this study adds}

- This large sample of 'treated glaucoma and $\mathrm{OHT}^{\prime}$ patients in secondary care demonstrated that the majority of VF progression occurs at moderate rates ( -0.3 to $-2 \mathrm{~dB}$ /year). Furthermore, these moderate progressors constitute the majority of those who ended with visual loss in their better eye.

- With increasing life expectancy, these moderate progressors may become increasingly clinically important.

- Those patients with initial VF damage are much more likely to develop a significant visual disability during their follow-up.

- Emphasis should be placed on identifying those patients with early VF defects and ensuring they are appropriately monitored.

\section{Conflict of interest}

The authors declare no conflict of interest. 


\section{Acknowledgements}

We would like to thank Neil Shumsky and David Johnston of Medisoft for their help in extracting the VF data for this study.

\section{References}

1 European Glaucoma Society. Terminology and Guidelines for Glaucoma. 3rd edn, 2008; European Glaucoma Society: Savona, Italy.

2 Chauhan BC, Garway-Heath DF, Goñi FJ, Rossetti L, Bengtsson B, Viswanathan AC et al. Practical recommendations for measuring rates of visual field change in glaucoma. Br J Ophthalmol 2008; 92(4): 569-573.

3 Heijl A. Computerized perimetry in glaucoma management. Acta Ophthalmol (Copenh) 1989; 67(1): 1-12.

4 Heijl A, Buchholz P, Norrgren G, Bengtsson B. Rates of visual field progression in clinical glaucoma care. Acta Ophthalmol 2013; 91(5): 406-412.

5 Chauhan BC, Mikelberg FS, Balaszi AG, LeBlanc RP, Lesk MR, Trope GE et al. Canadian Glaucoma Study: 2. risk factors for the progression of open-angle glaucoma. Arch Ophthalmol 2008; 126(8): 1030-1036.

6 De Moraes CG, Liebmann JM, Liebmann CA, Susanna Jr R, Tello C, Ritch R. Visual field progression outcomes in glaucoma subtypes. Acta Ophthalmol 2013; 91(3): 288-293.

7 Demirel S, De Moraes CG, Gardiner SK, Liebmann JM, Cioffi GA, Ritch R et al. The rate of visual field change in the ocular hypertension treatment study. Invest Ophthalmol Vis Sci 2012; 53(1): 224-227.

8 Forchheimer I, de Moraes CG, Teng CC, Folgar F, Tello C, Ritch $\mathrm{R}$ et al. Baseline mean deviation and rates of visual field change in treated glaucoma patients. Eye (Lond) 2011; 25(5): 626-632.

9 Pereira ML, Kim CS, Zimmerman MB, Alward WL, Hayreh SS, Kwon YH. Rate and pattern of visual field decline in primary open-angle glaucoma. Ophthalmology 2002; 109(12): 2232-2240.

10 Lockwood AJ, Kirwan JF, Ashleigh Z. Optometrists referrals for glaucoma assessment: a prospective survey of clinical data and outcomes. Eye (Lond) 2010; 24(9): 1515-1519.

11 Arnalich-Montiel F, Casas-Llera P, Muñoz-Negrete FJ, Rebolleda G. Performance of glaucoma progression analysis software in a glaucoma population. Graefes Arch Clin Exp Ophthalmol 2009; 247(3): 391-397.

12 Casas-Llera P, Rebolleda G, Muñoz-Negrete FJ, Arnalich-Montiel F, Pérez-López M, Fernández-Buenaga R. Visual field index rate and event-based glaucoma progression analysis: comparison in a glaucoma population. Br J Ophthalmol 2009; 93(12): 1576-1579.

13 Fitzke FW, Hitchings RA, Poinoosawmy D, McNaught AI, Crabb DP. Analysis of visual field progression in glaucoma. Br J Ophthalmol 1996; 80(1): 40-48.

14 Wesselink C, Heeg GP, Jansonius NM. Glaucoma monitoring in a clinical setting: glaucoma progression analysis vs nonparametric progression analysis in the Groningen Longitudinal Glaucoma Study. Arch Ophthalmol 2009; 127(3): 270-274.

15 Bengtsson B, Heijl A. Evaluation of a new perimetric threshold strategy, SITA, in patients with manifest and suspect glaucoma. Acta Ophthalmol Scand 1998; 76(3): 268-272.
16 Brenton RS, Phelps CD. The normal visual field on the Humphrey field analyzer. Ophthalmologica 1986; 193(1-2): 56-74.

17 Olsson J, Asman P, Heijl A. A perimetric learner's index. Acta Ophthalmol Scand 1997; 75(6): 665-668.

18 Saunders LJ, Russell RA, Crabb DP. Practical landmarks for visual field disability in glaucoma. Br J Ophthalmol 2012; 96(9): 1185-1189.

19 Thygesen J, Aagren M, Arnavielle S, Bron A, Fröhlich SJ, Baggesen $\mathrm{K}$ et al. Late-stage, primary open-angle glaucoma in Europe: social and health care maintenance costs and quality of life of patients from 4 countries. Curr Med Res Opin 2008; 24(6): 1763-1770.

20 Heijl A, Bengtsson B. The effect of perimetric experience in patients with glaucoma. Arch Ophthalmol 1996; 114(1): 19-22.

21 De Moraes CG, Sehi M, Greenfield DS, Chung YS, Ritch R, Liebmann JM. A validated risk calculator to assess risk and rate of visual field progression in treated glaucoma patients. Invest Ophthalmol Vis Sci 2012; 53(6): 2702-2707.

22 Toh S, Platt R. Big data in epidemiology: too big to fail? Epidemiology 2013; 24(6): 939

23 Ang GS, Shunmugam M, Azuara-Blanco A. Effect of cataract extraction on the glaucoma progression index (GPI) in glaucoma patients. J Glaucoma 2010; 19(4): 275-278.

24 Heijl A, Bengtsson B, Hyman L, Leske MC. Early Manifest Glaucoma Trial Group. Natural history of open-angle glaucoma. Ophthalmology 2009; 116(12): 2271-2276.

25 Trikha S, Macgregor C, Jeffery M, Kirwan J. The Portsmouth-based glaucoma refinement scheme: a role for virtual clinics in the future? Eye (Lond) 2012; 26(10): 1288-1294

26 Anderson DR, Drance SM, Schulzer M. Natural history of normal-tension glaucoma. Ophthalmology 2001; 108(2): 247-253.

27 Chen PP. Blindness in patients with treated open-angle glaucoma. Ophthalmology 2003; 110(4): 726-733.

28 De Moraes CG, Juthani VJ, Liebmann JM, Teng CC, Tello C, Susanna Jr R. Risk factors for visual field progression in treated glaucoma. Arch Ophthalmol 2011; 129(5): 562-568

29 Eid TM, Spaeth GL, Bitterman A, Steinmann WC. Rate and amount of visual loss in 102 patients with open-angle glaucoma followed up for at least 15 years. Ophthalmology 2003; 110(5): 900-907.

30 Kwon YH, Kim CS, Zimmerman MB, Alward WL, Hayreh SS. Rate of visual field loss and long-term visual outcome in primary open-angle glaucoma. Am J Ophthalmol 2001; 132(1): 47-56.

31 Nouri-Mahdavi K, Zarei R, Caprioli J. Influence of visual field testing frequency on detection of glaucoma progression with trend analyses. Arch Ophthalmol 2011; 129(12): 1521-1527.

32 Crabb DP, Garway-Heath DF. Intervals between visual field tests when monitoring the glaucomatous patient: wait-and-see approach. Invest Ophthalmol Vis Sci 2012; 53(6): 2770-2776.

33 Rao HL, Kumar AU, Babu JG, Senthil S, Garudadri CS. Relationship between severity of visual field loss at presentation and rate of visual field progression in glaucoma. Ophthalmology 2011; 118(2): 249-253.

34 Malik R, Baker H, Russell RA, Crabb DP. A survey of attitudes of glaucoma subspecialists in England and Wales to visual field test intervals in relation to NICE guidelines. BMJ Open 2013; 3(5): e002067. 